\title{
3. JOHANNES HOLUBARZ, INFORMATOR SERENISSIMI PRINCIPIS
}

Der folgende Abschnitt nützt die seltene Gelegenheit, den Erzieher eines spätmittelalterlichen Habsburgerregenten als zeittypisch vielseitige und bemerkenswerte Persönlichkeit hervortreten zu lassen. Eine zusätzliche Facette dabei ist, daß wir auch über das nicht weniger abenteuerliche, aber tragische curriculum von Kaspar Wendel, dem „fridericianischen" Präceptor von Ladislaus Postumus relativ gut Bescheid wissen" ${ }^{93}$. Für den ersten böhmischen Lebensabschnitt Johannes Holubařs kann ich mich auf die sehr ausgewogenen Forschungen Zahradniks stützen ${ }^{94}$.

Die früheste verwertbare Nennung in einer Urkunde des Prager Nationalmuseums weist Holubař als katholisch aus, was sich in seinem späteren Lebensweg ja bestätigt hat: Johannes dictus Holubars senior de Nachod, clericus Pragensis diocesis ist am 21. Februar 1453 in Wien anwesend. Ein Magister Lucas Schenck de Brunna, baccalaureus in decretis constituit eum procuratorem et nuncium, wobei nochmals vom dictum Johannem Holubars geschrieben wird. Nun nimmt Zahradnik wohl mit Recht an, daß dieser Kleriker Holubars sich - im Zusammenhang mit der Herausgabe des Prinzen Ladislaus durch Friedrich III. im Jahr 1452 - im Gefolge des böhmischen Thronerben befand. Ladislaus Postumus dürfte also schon vor seinen zeitweiligen Aufenthalten in Böhmen tschechisch gelernt haben.

Diese Vermutung wird nach meinen Recherchen fast zur Gewißheit angesichts der quellenmäßig belegbaren engen Bindung Holubařs an den einflußreichen Grafen Ulrich von Cilli, der den Kronprinzen Ladislaus vor den Toren Wiener Neustadts übernommen hatte. Dies geschah bekanntlich nach einer symbolischen Reinwaschung, damit „....wenn noch etwas Steirisches an ihm haften geblieben, er es gänzlich abtäte" ${ }^{95}$. Von Wien begab sich Holubař mit dem künftigen König nach Prag und gab - weil vermutlich noch durch keine Weihe gebunden - das Vorhaben, Priester zu werden auf, es wäre nicht die letzte Volte in seiner Biographie gewesen. Er widmete sich fortan ganz der Aufgabe als königlicher informator und - wie wir aufgrund eines gleich zu besprechenden Ereignisses annehmen dürfen - auch als Leibwächter und vielleicht Turnierlehrer. Allenfalls läßt das Beiwort senior der ersten Nennung aber auch an einen gleichnamigen nahen Verwandten des Prinzenerziehers denken. Aus einem Krönungsbericht Aeneas Silvius Piccolominis an Papst Nikolaus V. erfahren wir übrigens, daß Ladislaus ein Jahr später noch nicht fließend Tschechisch konnte ${ }^{96}$.

93 Vgl. K. Grossmann, Die Frühzeit des Humanismus in Wien bis zu Celtis Berufung 1497. Jahrbuch für Landeskunde von Niederösterreich N. F. 22 (1929) 196-197. L. Brunner, Eggenburg. Geschichte einer niederösterreichischen Stadt 1. Eggenburg 1933, besonders 194-196.

94 Zahradnik, Slovník (zit. A. 7) 12-15.

95 Zur Entlassung Ladislaus aus der „Gerhabschaft“ Friedrichs III. vgl. G. ButTlar, Die Belagerung des Ladislaus Postumus in Wiener Neustadt von 1452 (Militärhistorische Schriftenreihe 57). Wien 1986, 26-27. Zum Einzug in Wien s. zuletzt F. OpLL, Leben im mittelalterlichen Wien. Wien-Köln-Weimar 1998, 124-125.

96 Volunt Bohemi regem suum apud se manere, donec patrium sermonem ediscat regnicolisque sine interprete loquatur, s. B. Widmer, Enea Silvio Piccolomini. Papst Pius II. Ausgewählte Texte aus seinen Schriften. Basel-Stuttgart 1960, 222-223. 
Die böhmischen Hoflehentafeln lassen erkennen, daß er sich gegenüber Holubar̆ vor allem im Jahr 1454 als König erkenntlich zeigte. Am 24. Juni verbrieft er ihm eine jährliche Lebensrente von 30 hriven in der Stadt Olmütz, am 6. Oktober folgt eine Auszahlung von 300 hřven $^{97}$. Am Samstag vor St. Veit schenkt er Racek von Vyškov und seinem Gefährten Jan Holubarz von Nachod die Saazer Ortschaften Chotěbytice (Kettowitz) und Mladějov pro serviciis eorum. Dem folgt am Donnerstag nach St. Stanislaus eine weitere Schenkung im Leitmeritzer Kreis. Schließlich überschreibt Ladislaus 1455, am Tag des heiligen Nikolaus, Holubař pro serviciis suis noch das Dorf Nemyčeves bei Jičin.

Außerdem dürfte Holubař - vielleicht von Georg Podiebrad oder Ladislaus, eher von Friedrich III., als dessen (Hof)rat er ja genannt ist - in den Adelsstand erhoben worden sein. Das in frühen Urkunden häufige de Nachod dürfte eine reine Herkunftsangabe sein ${ }^{98}$. Andernfalls hätte es der informator serenissimi principis seiner bei aller Demutshaltung doch auf Repräsentation ausgelegten Darstellung auf $3^{\mathrm{r}}$ des Ladislauskodex nicht vorenthalten (vgl. Abb. 5) und auch in den späteren österreichischen Urkunden verwendet.

Für die weitere Biographie müssen wir einen Sprung machen: Der vorhin genannte Ulrich II. von Cilli war Besitzer der Herrschaft Liechtenstein in Maria Enzersdorf bei Wien und berief um 1454/55 die Franziskaner, was von den benachbarten Pfarrherren Thomas Ebendorfer in Perchtoldsdorf bzw. Johann von Hinderbach in Mödling aus Konkurrenzgründen wohl mehr als nur beargwöhnt wurde. Der charismatische Generalvikar Giovanni da Capestrano war sogar willens, die Brüder aus Maria Enzersdorf wieder abzuziehen. Erst Ulrichs Versprechen, alle Schwierigkeiten aus dem Weg zu räumen und die Niederlassung entsprechend zu fundieren, rettete das Observantenkloster ${ }^{99}$.

Zuvor aber zog der Cillier mit einem vom ungarischen Reichsverweser Johann Hunyadi geführten Kreuzheer, begleitet vom bereits genannten italienischen Feldprediger Capestrano, gegen die Türken. Beim Entsatz von Belgrad ließen die beiden Hunyadisöhne, die den listigen Mentor von Ladislaus als politischen Konkurrenten fürchteten, das königliche Heer in die Festung und verschlossen die Tore. Daraufhin luden sie Ulrich zu einer Unterredung und ließen ihn am 9. 11. 1456 erschlagen. Diesen Mord und die nachfolgende Rettung des Königs Ladislaus durch Johannes Holubař und einige andere böhmische Ritter schildert Michel Beheim, 1455/56 im Dienste von Ladislaus Postumus und daher zumindest Ohrenzeuge, in seinem Gedicht Von dem Turken Keiser Machamet, wie er Constantinopel gewan, Vers 10031008:

$$
\begin{aligned}
& \text { Pei dem der jung von Krei } \\
& \text { und der her von Vetan } \\
& \text { und her Jan von Katscha } \\
& \text { her Jan Halub, Her Gybisch } \\
& \text { wider die schelk vil dibisch } \\
& \text { stalten sie sich zur wer }{ }^{100} \text {. }
\end{aligned}
$$

97 Archiv český čili staré písemné památký české í moravské, sebrané z archivu domácích í cizích. VII. Prag 1887, 582-583.

98 Zahradnik, Slovník (zit. A.7) 13-15 diskutiert das Problem de Nachod ausführlich.

99 Vgl. dazu J. Hofer, Johannes Kapistran. Ein Leben für die Reform der Kirche II (Bibliotheca Franciscana 2). Neue, bearb. Aufl. Heidelberg 1965, 326 und S. Petrin, Geschichte von Maria Enzersdorf. Wien 1979,22 und 59.

100 Die Gedichte des Michel Beheim 2. Hrsg. von H. Gilde und Ingeborg Spriewald (Deutsche Texte des Mittelalters 64) Berlin 1970, 703. 
Diese Szene mit Jan Halub und ein noch folgender Turnierbericht belegen Holubařs Wehrhaftigkeit und lassen - im Zusammenhang mit den „leitmotivischen“ bewaffneten Knaben und seiner martialischen Selbstdarstellung im Cod. Pal. lat. 1787, die indirekt auf seine Lebensrettung des Königs hinweisen könnte - sogar eine Tätigkeit als Fecht- und Turnierlehrer des jungen Ladislaus Postumus vermuten. Erwähnenswert ist unter diesem Gesichtspunkt, daß der berühmte Elfenbeinsattel von 1456 für Ladislaus ein gekröntes Monogramm V (für Vladislav) enthält, das zu den Buchstaben des Tücheralphabets in Holubařs Vokabular paßt, in dem gerade das $V$ fehlt. (vgl. Abb. 35 gegen Abb.10) ${ }^{101}$. Ein unmittelbares Vorbild dafür könnte der Prunksattel für Wenzel IV. mit Tüchermustern und entsprechender Monogrammierung gewesen sein ${ }^{102}$. Abschließend sei erwähnt, daß die Geschehnisse von Belgrad - allerdings ohne die Person Holubařs - im madjarischen Kulturbereich wohlbekannt sind, weil sie die Exposition der ersten ungarischen Nationaloper Hunyadi László (1844) von Ferencz Erkel bilden.

Wie eng das Verhältnis zwischen Holubař und Ulrich von Cilli gewesen war, beweist sich daran, daß der böhmische Ritter - ganz im Sinn des Verstorbenen - fortan das Franziskanerkloster in Maria Enzersdorf großzügig unterstützte und 1465/66 einen Neubau ermöglichte, woran übrigens eine die Baugeschichte zusammenfassende Tafel in der heutigen barocken Pfarrkirche erinnert. Holubař engagierte sich wohl auch deshalb, weil er nach dem Tod des letzten Cilliers sich in Maria Enzersdorf niederließ und Margret die Lichtenhoferin, Witwe des ehemaligen Pflegers der Herrschaft Liechtenstein heiratete. Sie hatte 1456 Ulrich von Cilli als Besitzerin der Burg Liechtenstein abgelöst ${ }^{103}$. Daraus erklärt sich auch die Verwunderung Zahradniks darüber, daß ab 1455 die Spuren Holubařs in böhmischen Quellen abrupt abbrechen ${ }^{104}$. Eine Wiener Urkunde vom 10. Dezember dieses Jahres aber bezeugt sowohl seinen Einfluß auf Ladislaus als auch seine alte Bindung an den Cillier: Ein auf Intervention von Freunden des böhmischen Königs Begnadigter schwört der Stadt Wien Urfehde. Besiegelt ist das Schriftstück von hern hern Hannsen von Don, des hern von Cili Haubtman und von Jann Holubarsky ${ }^{105}$. Nach dem Ende des Königs Ladislaus ist der soeben Genannte zunächst Pfleger von Marchegg. Von dort bezieht sich Hanns von Holuwersy, haubtman zu Marchegk in einem Brief an die Stadt Wien vom 19. April 1459 auf sein Schreiben an Friedrich III., ihn mit Rücksicht auf seine dem Land geleisteten Dienste bei seinen briefen und gerechtigkait zu belassen ${ }^{106}$. Allerdings läßt eine Wiener Urkunde Friedrichs III. vom

101 B. Thomas - O. Gamber, Katalog der Leibrüstkammer, 1. Der Zeitraum von 500 bis 1530 (Führer durch das Kunsthistorische Museum 13) Wien 1976, 70-71. Ein schönes Beispiel für „Bänderbuchstaben“ ist u.a. auch das Christogramm einers spätgotischen Tellers im Stift Zwettl, abgebildet bei EngLisch JARITZ, Das tägliche Leben (zit. A. 87) 78.

102 J. von Schlosser, Elfenbeinsättel des ausgehenden Mittelalters (JKhSW 15) Wien 1894, 260-262: Das Schärpenmotiv hängt gewiß mit der Turnierbahn zusammen. Vgl. das $r$ in Fig. 2, dazu die wenig späteren Bänderbuchstaben a und b auf einem heute in Florenz aufbewahrten Sattel wohl aus einer oberdeutschen Werkstatt, s. S. 277.

103 Vgl. Petrin, Maria Enzersdorf (zit. A. 99) 59; R. Büttner, Burgen und Schlösser zwischen Wienerwald und Leitha (Niederösterreich 1,1) Wien 1966, 150.

104 S. Zahradnik, Slovník (zit. A. 7) 13. Ein Prager Rechtsgeschäft betreffend einen Johannem Holub ist für 1466 belegt, s. V. V. Tomek, Základy I (zit. A. 71) 124.

105 Quellen zur Geschichte der Stadt Wien II/2, bearb. von K. Uhlirz. Wien 1900, Nr.3652. Die hier und in anderen Quellen zitierten Schreibungen des Familiennamens unseres Protagonisten kennzeichnen paradigmatisch die Variationsmöglichkeiten im zeitgenössischen (deutschen) Schreibusus.

106 Quellen zur Geschichte der Stadt Wien II/3, bearb. von K. Uhlinz. Wien 1904, Nr. 3877. 
19. März 1460 ... von der sprüch und vordrung wegen so ... Hanns Holobersy und Margaret s(ein) Hausfrau zu vns ... vermaintten gehabt ze haben ... die Möglichkeit offen, daß die Marchegger Pflegschaft nicht rechtmäßig bestand ${ }^{107}$. Schon am 17. November 1458 hatte Ladislaus, Graf von St. Georgen und Bösing (heute Pezinok in der Slowakei), dem Bürgermeister und Rat von Wien mitgeteilt, er habe sich schon fast vor Jahresfrist bei Herzog Albrecht über herrn Hannsen Holebarzy, phleger auf Marchegk beschwert, der seinen Holden das Vieh widerrechtlich genommen habe ${ }^{108}$. Es besteht also kaum ein Zweifel, daß Holubař in dieser unruhigen Zeit mit tschechischen Söldnerführern zumindest paktierte und als einer der ...höchst übel beleumundeten tschechischen Condottieri... ${ }^{109}$ eingeschätzt wurde. Holubař konnte es sich in diesem Zusammenhang sogar leisten, Kaiser Friedrich III. zu erpressen, wie der Eggenburger Ausgleich vom 16. Juni 1465 ausweist ${ }^{110}$.

Umgekehrt war er 1464 Mitstreiter der Kaiserlichen bei der Belagerung der Feste Urschendorf bei Wiener Neustadt gewesen, denn in Michel Beheims Buch von den Wienern scheint her hans halawersy zweimal bei Etlicher namen, dy uor uessendorf lagen auf ${ }^{111}$. In jedem Fall erwarb sich der frühere informator regis 1458 bis 1476 im Dienste Erzherzog Albrechts und seines kaiserlichen Bruders, aber auch Herzog Ludwigs des Reichen von Niederbayern Namen und Vermögen ${ }^{112} 1469$ führte er - mit wenig Erfolg - ein kaiserliches Heer gegen die Fronde der steirischen Aufrührer unter Andreas Baumkirchner an. Jakob Unrest berichtet in seiner Österreichischen Chronik diesbezüglich von ... Hollupp, ein Pehaym und des hertzog von Payren veldthawbtman. Holubař, oberster Hauptmann und kaiserlicher Rat $^{113}$ wird verwundet und nach Graz gebracht.

Von 1458 bis 1478 war der ehemalige Prinzenerzieher Pfleger der Herrschaft Liechtenstein, danach kaufte er das Schloß Wildegg, das er bis 1486 innehatte ${ }^{114}$. 1475 unterstützt ihn der Wiener Stadtrat im Zusammenhang mit der Einnahme der Feste Perchtoldsdorf,

107 E. Birk, Urkunden-Auszüge zur Geschichte Kaiser Friedrichs des III. in den Jahren 1452-1467. AfÖG 10 (1853) 234. Schließlich werden die Veste Marchegg sowie die Ungelter von Groß-Enzersdorf und Groß-Schweinbarth um 2000 Gulden ausgelöst, s. J. Chyel, Regesta chronologico-diplomatica Friderici IV. romanorum regis. Wien 1838, Nr.3820.

108 Quellen zur Geschichte der Stadt Wien II/2 (zit. A. 105) Nr. 3824.

109 K. Schalk, Mödlinger Grundbücher aus dem XV. Jahrhundert. Jahrbuch für Landeskunde von Niederösterreich 10 (1911) 187 A.1.

110 J. Chmer, Regesta chronologico-diplomatica Friderici IV. romanorum regis. Auszug aus den im k. k. geheimen Hofarchive zu Wien sich befindenden Reichsregisterbüchern vom Jahre 1440-1493. Wien 1838 (Nachdr. Hildesheim 1962) Anhang Nr. 129. Vgl. dazu L. Brunner, Eggenburg.Geschichte einer niederösterreichischen Stadt. Eggenburg 1933, $208 \mathrm{f}$.

111 Michael Beheim's Buch von den Wienern 1462-1465. Zum ersten Mahle hrsg. von Th. G. von Karajan. Wien $1843,373 / 14$ und 23.

112 S. F. Palack Ý, Geschichte der Böhmen 4/1 (zit. A. 6) 516; P. J. Heinig, Kaiser Friedrich III. (14401493). Hof, Regierung und Politik 1 (Forschungen zur Kaiser- und Papstgeschichte des Mittelalters. Beih. zu J. F. Böhmers Regesta Imperii 17) Köln-Weimar-Wien 1997, 428-430.

113 Urkunden und Actenstücke zur österreichischen Geschichte im Zeitalter Kaiser Friedrichs III. und König Georgs von Böhmen (1440-91) hrsg. von A. Bachmann (FRA Dipl. 42). Wien 1879, Nr. 468; Jakob Unrest, Österreichische Chronik. Hrsg. von K. Grossmann (MGH Script. Rer. Germ. N.S. 11) Weimar 1957, 26 ff.; J. Chyel, Regesta Friderici IV. (zit. A.100) Nr. 5777. Zu diesen Quellen vgl. ferner R. Schäffer, Die Baumkirchnerfehde (1469-71) in: Andreas Baumkirchner und seine Zeit (= Wissenschaftliche Arbeiten aus dem Burgenland 67) Eisenstadt 1983, besonders 159-160 und HeiniG, Friedrich III. (zit. A. 112) 391-392.

114 Vgl. R. K. Donin, Wildegg. Wien 1927, 15-16; S. Petrin, Maria Enzersdorf (zit. A. 99) 5. 
indem er Söldner nach Merkenstein schickt. Dazu melden die Stadtkämmererrechnungen ferner: Als herr hanns Holowersy Berchtolzdorf Ingenommen, hat man den Trummettern gelihen 2 Roß 80 (pfennig) ${ }^{115}$. Übrigens ist Holubař zwischen 1467 und 1481 in Perchtoldsdorf, dem ersten Aufenthaltsort Ladislaus nach seiner „Befreiung“ durch Ulrich von Cilli, als Besitzer von Äckern, Weingärten, einer Mühle und zwei Brotbänken belegbar ${ }^{116}$, und nur sehr mählich dürfte sich der Haudegen und Kriegsgewinnler zum friedlichen Gutsbesitzer gewandelt haben. Eine Wiener Urkunde vom 19. Mai 1464 belegt die Vermittlungsrolle des edeln vesten ritter hern Hannsen Holubersy in einer Fehde mit einem Traiskirchner Adeligen, eine Vösendorfer Urkunde vom 1483 handelt von einem Gewaltbrief auf das Erbe, den Herr Hanns Holwers zu Wildegk seinem Schwager dem Stüxenhauser nach dem Tod des Bruders ausstellt ${ }^{117}$ . Ein unruhiger Geist freilich blieb er: Am 10 Jänner 1472 stellt Paulus von Melk, Rektor der Wiener Universität, Frau Margaretha Holowersin eine Urkunde über die vor dem Universitätsgericht unter Eid abgelegte Stellungnahme des Studenten Berchtold Gebhart von Altorff aus, der von ihrem Mann Hanus Holowers zu einer Aussage über angeblichen sträflichen Umgang mit ihr gezwungen worden war ${ }^{118}$.

Fast alle späteren Dokumente nehmen Bezug auf eine Rechtshandlung, die Holubař als sehr reichen Mann mit erstklassiger Reputation ausweisen: Am 8. Dezember 1475 stellt Wiens Bürgermeister dem edln vesten rittern hern Hannsen Holowärsy einen Schuldbrief über 1000 guter Ungrischer guldein aus und verpflichtet sich zur Rückzahlung binnen Jahresfrist ${ }^{119}$. Davon wurden, wie Hanns Holubersy auf Johannstein bestätigt, am 23. November 1478 nur 300 Gulden zurückgekommen ${ }^{120}$. Doch er lieh der Stadt Wien offensichtlich weiter, denn am 9. April 1487 stellte Hanusch Holowarsch in der Prager Burg wegen seines haubtbriefs über 1000 ungarische Gulden und andere Beträge, die er dem Bürgermeister und Rat von Wien geliehen hatte, hern Jhanen Sokolowsky von Wraucz, kuniglicher majestat zu Beheim camrer eine Vollmacht aus ${ }^{121}$, was auch den Schluß zuläßt, daß sich Holubař nach dem Verkauf von Wildegg wieder nach Böhmen zurückzog.

Es verwundert nicht, daß bereits am 23. Mai 1487 König Matthias Corvinus von Ödenburg aus auf die Rückzahlung der Schuld an Jan Zokolowsky laut Kontrakt mit Hanns Holubarzi drängt ${ }^{122}$, weil der Letztgenannte auch zum mächtigen Ungarnkönig enge Beziehungen gehabt haben muß. Ein diesbezüglicher Bericht des hungaro-italienischen Geschichtsschreibers Marco Galeoti, der 1461 nach Ofen kam, ist von besonderem Wert, weil darin auch eine kurze Charakteristik unseres Ritters gegeben wird: Venerat Budam quidam, strenuissimus eques, natione Germanus, cognomine Holubar, virium et corporis mole mirabilis, qui in eo certamine, quod praetentis hastis equitum concursu fit, ferebatur invictus, plurimos enim secum certantes ad terram praetento conto prostraverat. An anderer Stelle heißt es nochmals ... cum

115 J. E. Schlager, Wiener Skizzen des Mittelalters N.F.III, 205-206, 260. Vgl. dazu S. Petrin, Perchtoldsdorf im Mittelalter. Forschungen zur Landeskunde von Niederösterreich 18. Wien 1969, 19 und 374, A. 85 .

116 Ebenda, 19 und 374, A. 87. Im Zusammenhang von Interesse ist das ladislausgerechte Wappenprogramm mit den Insignien des Drachenordens im Südportal der Perchtoldsdorfer Pfarrkirche, vgl. wieder Petrin, 15. Ab 1448/49 hatte Friedrich III. die Burg Perchtoldsdorf an Ulrich von Cilli verpfändet.

117 Quellen zur Geschichte der Stadt Wien II/3 (zit. A. 96) Nr. 4082 und 4407.

118 Ebenda, Nr.4968.

119 Ebenda, Nr. 4568.

120 Ebenda, Nr. 4708.

121 Ebenda, Nr. 5192.

${ }^{122}$ Quellen zur Geschichte der Stadt Wien II/3 (zit. A. 106), Nr. 5203. 
Holubar grandi corpore... In Buda fordert Matthias Corvinus ... fama His commotus ... Holubar̆ zum Turnierkampf auf. Der Ritter will diese Aufforderung zunächst nicht annehmen und entschließt sich, den König weitestgehend zu schonen. Daraufhin droht ihm der Corvine mit dem Tode, falls nicht rücksichtslos gefochten würde. Der Kampf ... in platea enim Divi Sigismundi ...findet statt. Galeoti... me quoque spectante... ist Zeuge der folgenden Szene, in der Holubař, mit einem Schlag auf die Stirn aus dem Sattel gehoben, sich die Hand bricht. Der ungarische König leistet daraufhin dem verletzten Ritter aufmerksamste Pflege und beschenkt ihn reichlich mit Pferden, teuren Gewändern und Geld ${ }^{123}$. Noch 1486 hat Matthias Corvinus Holubař als seinen Pfleger zu Liechtenstein erwähnt ${ }^{124}$. Diese Turnierszene wurde im neunzehnten Jahrhundert von namhaften Künstlern aufgegriffen und national interpretiert: Jan Kollár erwähnt den Ritter Holubař bei den Erläuterungen zum Lied Stála bitka, stála za Štefana krála (Unter König Stefan hat sich ein Gefecht zugetragen), wobei er unter Bezug auf Galeoti bemängelte, daß die lateinischen Geschichtsschreiber oft auch Tschechen sowie andere getaufte Slawen als Deutsche bezeichnen ${ }^{125}$. Der ungarische Historienmaler Sándor Wagner dagegen verherrlichte den Turniersieg der madjarischen Identifikationsfigur Matthias Corvinus über den kampfberühmten Böhmen. Sein Großgemälde für den Hauptsaal der 1864 neu eröffneten Pester Redoute (Fövárosi vigadó) wurde im Zweiten Weltkrieg vernichtet (vgl. Abb. 33) ${ }^{126}$.

Ganz im Sinn Sándor Wagners wurde die Turnierszene auch in der ungarischen Literatur verwertet. So läßt Miklós Jósika, einer der ersten bedeutenden ungarischen Romanciers, Holubař in seinem Werk A csehek magyarországban (Die Tschechen in Ungarn) auftreten, und auch das Holubařgedicht Budai harcjátek des Romantikers Karoly Kisfaludy aus dem Jahr 1828 ist bis heute geläufig geblieben. Später hat sich noch Sándor Endrödi des Themas lyrisch angenommen. Den originellsten Beitrag zur Rezeption des böhmischen Helden indes liefert ein 1877 erstmals veröffentlichtes satirisches Epos von Károly Szathmáry de Péterfalva, das sich ausdrücklich auf den vorhin genannten Roman Jósikas beruft. Allerdings versucht Szathmáry Holubař, dem er den Vornamen Wenzel verpaßt, aus dem heldischen Schatten von Geschichte und Zeitgeist zu holen. Unter Berufung auf Don Quichotte und Ulrich von Lichtenstein formt er den tschechischen Söldnerführer zu einer grotesk-komischen Rittergestalt, die durch eine Kette auch galanter Abenteuer - bis hin zum Kampf mit dem Ungarnkönig - stolpert. Entsprechend karikaturistisch sind die fünf dazu bekannten Illustrationen von verschiedenen Künstlern geraten. Unser mit Berger signiertes Beispiel zeigt, wie der vom Turnier ramponierte Protagonist buchstäblich wieder zusammengeflickt wird (vgl. Abb. 34).

Doch zurück zu den Schulden der Stadt Wien an Holubař. Sie ermöglichen uns eine indirekte Fixierung des Todes vor dem 10. Mai 1500. Denn mit diesem Datum schreibt immerhin ein polnischer Bischof aus Leslau in Kujavien im Namen der Erben nach Johanni Holu-

${ }_{123}$ Martius Galeotus, Libellus elegans de egregie, sapienter, iocose dictis ac factis Mathiae Sereniss(imi) Vngariae Regis, ad inclytum Ducem Joannem eius Filium. Wien 1563, Cap. XIII. Egregie Factum.

124 R. BütTner, Burgen (zit. A. 103) 150.

125 I. Th. Zahradnik, Slovník (zit. A. 7) 11.

126 Zum Bild Wagners vgl. V. Fraknor, A Hunyadiak és a Jagellók kora (1440-1526) (A magyar nemzet története 4). Budapest 1896, 322 und 664. Vgl. ferner M. JósikA, A csehek magyarországban. Korrajz I. Mátyás király idejéböl 4. Pest 1857, S. 88-89 und 130-138; K. Kisfaludy, Minden munkái...szerkeszté Toldy Ferencz, Bd. 1, Pest 1859, 153-156. Mir zugänglich war ferner die zweite unveränderte Auflage von K. Száthmáry de Péterfalva,Vitéz Holubár Wenczel lovag története viggal-elegy szomoru hösköltemény vi énekben. Budapest 1887. Vgl. hier insbesondere S. 12. 
barz wegen der 1000 ungarischen Gulden ${ }^{127}$. Die Sache wird übrigens erst am 20. September 1515 (!) nach einer kräftigen Intervention Maximilians I. geregelt ${ }^{128}$.

Dieses Dazwischentreten auch des Kaisers kann nicht verwundern und eine persönliche Bekanntschaft der Beiden - schon in früher Zeit - ist nicht unwahrscheinlich. Wie die Kontakte allerdings liefen, bleibt ein Geheimnis. Wir können jetzt nur annehmen, dass der Habsburger in seiner ersten Kindheit zumindest indirekt vom informator serenissimi principis gelernt hat. Formale Charakteristika wie die Fraktur und das Tücheralphabet des Cod. Pal. lat. 1787 kehren in den Lehrbüchern Maximilians wieder, und der Wiener CVP 2945, eine Kopie des Holubařvokabulars, die im im nächsten Kapitel vorgestellt wird, war wohl für den Sprachunterricht des jungen Monarchen gedacht. Maximilian könnte aber auch einfach neugierig auf den Praeceptor des verstorbenen Verwandten gewesen sein, der seine eigene Erziehung beeinflußt hatte ${ }^{129}$. Darüberhinaus dürfte den „letzten Ritter“, der einen Sinn für Außergewöhnliches hatte, diese Persönlichkeit mit ihrem imponierenden Äußeren, dem Nimbus eines Haudegens, Turnierkämpfers und Königsretters, dem Aufsteigerimage und nicht zuletzt den gesellschaftlichen Kontakten angezogen haben. Ein weiteres Argument ist politischer Natur: Holubař war mit dem kaiserlichen Vater Friedrich in Kontakt gestanden und besaß bis mindestens 1486 im südlichen Wienerwald eine nicht zu übergehende Machtkonzentration zwischen den Residenzen Wien und Wiener Neustadt. Es steht zu hoffen, daß dies auch Gründe sein werden, sich mit dem zeitweiligen Herrn von Wildegg grandi corpore - als paradigmatischem Vertreter des so widersprüchlichen fünfzehnten Jahrhunderts - in Zukunft etwas eingehender zu befassen.

Sicher war Holubař ein skrupelloser Söldnerführer, Draufgänger und Spekulant. Er war aber ebenso ein vielseitiger Prinzenerzieher, einflußreicher Burgpfleger und verläßlicher Patronatsherr der Kirche. Sein Lehrbuch für Ladislaus zeigt ihn - kulturell wie ästhetisch - auf der Höhe der Zeit, die Verschränkungen mit dem Melk-Tepler Gebetbuch lassen weitere Zusammenhänge vermuten, vgl. S. 20 ff. Da das Todesjahr mit 1500 festliegt, hat Holubař - wie übrigens auch Kaiser Friedrich III. - ein für die Zeit fast biblisches Alter erreicht. Er übernahm sein Amt als Erzieher von Ladislaus vermutlich nach dessen Übergabe an die Stände 1452, dürfte damals also schon über zwanzig Jahre gewesen sein. Somit ergibt sich eine Lebensspanne zwischen siebzig und achtzig Jahren.

${ }_{127}$ Quellen zur Geschichte der Stadt Wien IV/1, bearb. von J. Lampel. Wien 1917, Nr. 5693.

128 Ebenda, Nr. 6079 und 6088.

129 Es ist im Zusammenhang bemerkenswert, daß Kaiser Maximilian im Jahr 1500 Weisung gab, in Ambras nach seinem wohl ersten Lehrbuch, dem Ladislausdonat (heute CVP $23^{\circ}$ der Wiener Nationalbibliothek) zu suchen, vgl. Fichtexau, Lehrbücher (zit. A. 19) 9. 